\title{
Banach contraction principle for cyclical mappings on partial metric spaces
}

\author{
T Abdeljawad ${ }^{1 *}$, JO Alzabut ${ }^{2}$, A Mukheimer ${ }^{2}$ and Y Zaidan ${ }^{2,3}$
}

\author{
"Correspondence: \\ thabet@cankaya.edu.tr \\ 1 Department of Mathematics, \\ Çankaya University, Ankara, 06530, \\ Turkey \\ Full list of author information is \\ available at the end of the article
}

\begin{abstract}
We prove that the Banacah contraction principle proved by Matthews in 1994 on 0 -complete partial metric spaces can be extended to cyclical mappings. However, the generalized contraction principle proved by (llić et al. in Appl. Math. Lett. 24:1326-1330, 2011) on complete partial metric spaces can not be extended for cyclical mappings. Some examples are given to illustrate our results. Moreover, our results generalize some of the results obtained by (Kirk et al. in Fixed Point Theory 4(1):79-89, 2003). An Edelstein type theorem is also extended when one of the sets in the cyclic decomposition is 0-compact.

MSC: $47 \mathrm{H} 10 ; 54 \mathrm{H} 25$
\end{abstract}

Keywords: partial metric space; fixed point; cyclic mapping; Banach contraction principle; 0-compact set

\section{Introduction and preliminaries}

The Banach contraction mapping principle is considered to be the core of many extended fixed point theorems. It has widespread applications in many branches of mathematics, engineering, and computer science. During the last decades many authors were able to generalize this principle [1-4]. After the appearance of partial metric spaces as a place for distinct research work into flow analysis, non-symmetric topology, and domain theory $[5,6]$, many authors started to generalize this principle to these spaces (see [7-14]). However, the contraction type conditions used in those generalizations do not reflect the structure of a partial metric space apparently. Later, the authors in [15] proved a more reasonable contraction principle in a partial metric space. The contraction type condition used there should logically be called a partial contractive condition. In this work, we show that the contraction principle obtained in [6] can be generalized to cyclical mappings. In contrast, the principle proved in [15] cannot be extended for a cyclical case. An Edelstein type theorem is also extended when one of the sets in the cyclic decomposition is 0 -compact. Some examples are also given to support our claims throughout the article.

A partial metric space (PMS) (see, e.g., $[5,6]$ ) is a pair $X, p: X \times X \rightarrow \mathbb{R}^{+}$, where $\mathbb{R}^{+}$ denotes the set of all nonnegative real numbers, such that

(P1) $p(x, y)=p(y, x)$ (symmetry)

(P2) If $0 \leq p(x, x)=p(x, y)=p(y, y)$ then $x=y$ (equality)

(P3) $p(x, x) \leq p(x, y)$ (small self-distances)

(P4) $p(x, z)+p(y, y) \leq p(x, y)+p(y, z)$ (triangularity)

for all $x, y, z \in X$.

\section{Springer}

( 2012 Abdeljawad et al.; licensee Springer. This is an Open Access article distributed under the terms of the Creative Commons Attribution License (http://creativecommons.org/licenses/by/2.0), which permits unrestricted use, distribution, and reproduction in any medium, provided the original work is properly cited. 
For a partial metric $p$ on $X$, the function $p^{s}: X \times X \rightarrow \mathbb{R}^{+}$given by

$$
p^{s}(x, y)=2 p(x, y)-p(x, x)-p(y, y)
$$

is a (usual) metric on $X$. Each partial metric $p$ on $X$ generates a $T_{0}$ topology $\tau_{p}$ on $X$ with a base of the family of open $p$-balls $\left\{B_{p}(x, \varepsilon): x \in X, \varepsilon>0\right\}$, where $B_{p}(x, \varepsilon)=\{y \in X: p(x, y)<$ $p(x, x)+\varepsilon\}$ for all $x \in X$ and $\varepsilon>0$.

Definition 1 (see, e.g., $[5,6,13])$

(i) A sequence $\left\{x_{n}\right\}$ in a PMS $(X, p)$ converges to $x \in X$ if and only if $p(x, x)=\lim _{n \rightarrow \infty} p\left(x, x_{n}\right)$.

(ii) A sequence $\left\{x_{n}\right\}$ in a PMS $(X, p)$ is called Cauchy if and only if $\lim _{n, m \rightarrow \infty} p\left(x_{n}, x_{m}\right)$ exists (and is finite).

(iii) A PMS $(X, p)$ is said to be complete if every Cauchy sequence $\left\{x_{n}\right\}$ in $X$ converges, with respect to $\tau_{p}$, to a point $x \in X$ such that $p(x, x)=\lim _{n, m \rightarrow \infty} p\left(x_{n}, x_{m}\right)$.

(iv) A mapping $f: X \rightarrow X$ is said to be continuous at $x_{0} \in X$, if for every $\varepsilon>0$, there exists $\delta>0$ such that $f\left(B_{p}\left(x_{0}, \delta\right)\right) \subset B_{p}\left(f\left(x_{0}\right), \varepsilon\right)$.

Lemma 2 (see, e.g., $[5,6,13])$

(A) A sequence $\left\{x_{n}\right\}$ is Cauchy in a PMS $(X, p)$ if and only if $\left\{x_{n}\right\}$ is Cauchy in a metric space $\left(X, p^{s}\right)$.

(B) A PMS $(X, p)$ is complete if and only if the metric space $\left(X, p^{s}\right)$ is complete. Moreover,

$$
\lim _{n \rightarrow \infty} p^{s}\left(x, x_{n}\right)=0 \Leftrightarrow p(x, x)=\lim _{n \rightarrow \infty} p\left(x, x_{n}\right)=\lim _{n, m \rightarrow \infty} p\left(x_{n}, x_{m}\right) .
$$

Lemma 3 Let $(X, p)$ be a partial metric space, and let $T: X \rightarrow X$ be a continuous selfmapping. Assume $\left\{x_{n}\right\} \in X$ such that $x_{n} \rightarrow z$ as $n \rightarrow \infty$. Then

$$
\lim _{n \rightarrow \infty} p\left(T x_{n}, T z\right)=p(T z, T z) .
$$

Proof Let $\epsilon>0$ be given. Since $T$ is continuous at $z$, find $\delta>0$ such that $T\left(B_{p}(z, \delta)\right) \subseteq$ $B_{p}(T z, \epsilon)$. Since $x_{n} \rightarrow z$, then $\lim _{n \rightarrow \infty} p\left(x_{n}, z\right)=p(z, z)$, and hence find $n_{0} \in N$ such that $p(z, z) \leq p\left(x_{n}, z\right)<p(z, z)+\delta$ for all $n \geq n_{0}$. That is $x_{n} \in B_{p}(z, \delta)$ for all $n \geq n_{0}$. Thus $T\left(x_{n}\right) \in$ $B_{p}(T z, \epsilon)$ and so $p(T z, T z) \leq p\left(T x_{n}, T z\right)<p(T z, T z)+\epsilon$ for all $n \geq n_{0}$. This shows our claim.

A sequence $\left\{x_{n}\right\}$ is called 0-Cauchy [16] if $\lim _{m, n \rightarrow \infty} p\left(x_{n}, x_{m}\right)=0$. The partial metric space $(X, p)$ is called 0 -complete if every 0 -Cauchy sequence in $x$ converges to a point $x \in X$ with respect to $p$ and $p(x, x)=0$. Clearly, every complete partial metric space is 0 -complete. The converse need not be true.

Example 4 (see [15]) Let $X=\mathbb{Q} \cap[0, \infty)$ with the partial metric $p(x, y)=\max \{x, y\}$. Then $(X, p)$ is a 0 -complete partial metric space which is not complete.

Theorem 5 ([6]) Let $(X, p)$ be a 0-complete partial metric space and $f: X \rightarrow X$ be such that

$$
p(f(x), f(y)) \leq \alpha p(x, y) \quad \forall x, y \in X \text { and } \alpha \in[0,1) .
$$


There exists a unique $u \in X$ such that $u=f(u)$ and $p(u, u)=0$.

Let $\rho_{p}=\inf \{p(x, y): x, y \in X\}$ and define $X_{p}=\left\{x \in X: p(x, x)=\rho_{p}\right\}$.

Theorem $6([15])$ Let $(X, p)$ be a complete metric space, $\alpha \in[0,1)$, and let $T: X \rightarrow X$ be a given mapping. Suppose that for each $x, y \in X$, the following condition holds:

$$
p(x, y) \leq \max \{\alpha p(x, y), p(x, x), p(y, y)\}
$$

Then

(1) the set $X_{p}$ is nonempty;

(2) there is a unique $u \in X_{p}$ such that $T u=u$;

(3) for each $x \in X_{p}$, the sequence $\left\{T^{n} x\right\}_{n \geq 1}$ converges, with respect to the metric $p^{s}$, to $u$.

Definition 7 Let $A$ and $B$ be two nonempty closed subsets of a complete partial metric space $(X, p)$ such that $X=A \cup B$. A mapping $T: X \rightarrow X$ is called a cyclical contraction if it satisfies:

(C1): $T(A) \subseteq B$ and $T(B) \subseteq A$.

(C2): There exists $0<\alpha<1: p(T x, T y) \leq \alpha p(x, y), \forall x \in A$ and $\forall y \in B$.

If (C2) in Definition 7 is replaced by the condition

(PC2): there exists $0<\alpha<1: p(T x, T y) \leq \max \{\alpha p(x, y), p(x, x), p(y, y)\} \forall x \in A$ and $\forall y \in B$, then $T$ is called a partial cyclical contraction. Note that partial cyclical contractions reflect the structure of a partial metric space better. The proof of the following lemma can be easily done by using the partial metric topology.

Lemma 8 A subset $A$ of a partial metric space is closed if and only if $x \in A$ whenever $x_{n} \in A$ satisfies $x_{n} \rightarrow x$.

Definition 9 A subset $A$ of a partial metric space $(X, p)$ is called 0-compact if, for any sequence $\left\{x_{n}\right\}$ in $A$, there exists a subsequence $\left\{x_{k_{n}}\right\}$ and $x \in A$ such that $\lim _{n \rightarrow \infty} p\left(x_{k_{n}}, x\right)=$ $p(x, x)=0$.

Clearly, a closed subset of a 0-compact set is 0 -compact.

Lemma 10 (see also [7] and [9]) Assume $x_{n} \rightarrow z$ as $n \rightarrow \infty$ in a PMS (X,p) such that $p(z, z)=0$. Then $\lim _{n \rightarrow \infty} p\left(x_{n}, y\right)=p(z, y)$ for every $y \in X$.

\section{Main results}

We start this section with a theorem that will motivate us to obtain our main result for cyclic contraction mappings.

Theorem 11 Let $(X, p)$ be a 0 -complete partial metric space and $T: X \rightarrow X$ be continuous such that

$$
p\left(T x, T^{2} x\right) \leq \alpha p(x, T x) \quad \forall x \in X, \text { where } \alpha \in(0,1) .
$$

Then there exists $z \in X$ such that $p(z, z)=0$ and $p(T z, z)=p(T z, T z)$. 
Proof The condition (3) implies that the sequence $T^{n}(x)$ is 0 -Cauchy for all $x \in X$. Hence, there exists $z \in X$ such that $x_{n}=T x_{n-1}$ converges to $z$ and $p(z, z)=0$. The conclusion that $p(T z, z)=p(T z, T z)$ follows by Lemma 3, (P2), and the inequality

$$
p(T z, z) \leq p\left(T z, x_{n+1}\right)+p\left(x_{n+1}, z\right) .
$$

Observe that if the partial metric in Theorem 11 is replaced by a metric, then we conclude that $z$ is a fixed point. The following theorem is an extension of Theorem 1.1 in [17].

Theorem 12 Let $A$ and $B$ be two nonempty closed subsets of a 0-complete partial metric space $(X, p)$ such that $X=A \cup B$, and suppose $T: X \rightarrow X$ is a cyclical contraction selfmapping of $X$. Then $T$ has a unique fixed point in $A \cap B$.

Proof The condition (C1) implies that for any $x \in A \cup B$,

$$
p\left(T x, T^{2} x\right) \leq \alpha p(x, T x)
$$

and this by (P4) implies that the sequence $\left\{T^{n}(x)\right\}$ is 0 -Cauchy for any $x \in X$. Consequently, $\left\{T^{n}(x)\right\}$ converges to some point $z \in X$ such that $p(z, z)=0$. However, in view of (C2), an infinite number of terms of the sequence $\left\{T^{n}(x)\right\}$ lie in $A$ and an infinite number of terms lie in $B$. Then by Lemma 8 , we conclude that $z \in A \cap B$, so $A \cap B \neq \emptyset$. Now (C1) and (C2) imply that the map $T$ restricted to $A \cap B$ is a contraction. Then the result follows by Theorem 5 .

We next give an example showing that the generalization to a partial metric space in Theorem 12 is proper.

Example 13 Let $X=[0,1], A=\left[0, \frac{1}{2}\right]$ and $B=\left[\frac{1}{2}, 1\right]$. Then $X=A \cup B$ and $A \cap B=\left\{\frac{1}{2}\right\}$. Provide $X$ with the partial metric $p(x, y)=|x-y|$ if both $x, y \in[0,1)$ and $p(x, y)=\max \{x, y\}$ otherwise. Then, clearly, $(X, p)$ is a complete partial metric space. Define $T: X \rightarrow X$ by $T(x)=\frac{1}{2}$ if $0 \leq x<1$ and $T(1)=0$. Then it can be easily checked that $T$ is a cyclical contraction with $\alpha=\frac{3}{4}$. Notice that the cyclical contractive condition of Theorem 12 is not satisfied when the partial metric $p$ is replaced by the usual absolute value metric.

The following example shows that Theorem 6 cannot be extended for cyclical mappings when the cyclical contraction is replaced by a partial cyclical contraction.

Example 14 Let $A=[0,1], B=[3,4] \cup\left\{\frac{3}{2}\right\}$ and $X=A \cup B$. Define $p: X \times X \rightarrow[0, \infty)$ by $p(x, y)=\max \{x, y\}$. Then $(X, p)$ is a complete partial metric space. Define $T: X \rightarrow X$ by

$$
T(x)= \begin{cases}\frac{3}{2}, & 0 \leq x \leq 1 \\ \frac{1}{2}, & x=\frac{3}{2} \\ \frac{x-2}{2}, & 3 \leq x \leq 4 .\end{cases}
$$

It can be easily seen that

$$
p(T x, T y)=\max \left\{\frac{3}{2}, \frac{y-2}{2}\right\}=\frac{3}{2} \leq \max \{\alpha p(x, y), p(x, x), p(y, y)\}=y,
$$

for any $x \in A, y \in B$ and any $\alpha \in(0,1)$. However, $A \cap B=\emptyset$. 
Corollary 15 Let $A$ and $B$ be two nonempty closed subsets of a complete partial metric space $(X, p)$ such that $X=A \cup B$. Let $f: A \rightarrow B$ and $g: B \rightarrow A$ be two functions such that $f(x)=g(x)$ for all $x \in A \cap B$ and

$$
p(f(x), g(y)) \leq \alpha p(x, y) \quad \forall x \in A \text { and } y \in B
$$

where $0<\alpha<1$. Then there exists a unique $x_{0} \in A \cap B$ such that

$$
f\left(x_{0}\right)=g\left(x_{0}\right)=x_{0}
$$

Proof Apply Theorem 12 to the mapping $T: A \cup B \rightarrow A \cup B$ defined by the setting

$$
T(x)= \begin{cases}f(x), & x \in A, \\ g(x), & x \in B .\end{cases}
$$

Observe that the assumption that $f(x)=g(x)$ for all $x \in A \cap B$ implies that $T$ is well defined.

Note that in the metric space case, the condition (4) implies that the map $T$ is well defined.

Obviously Theorem 12 can be extended to the following version.

Theorem 16 Let $\left\{A_{i}\right\}_{i=1}^{k}$ be nonempty closed subsets of a 0 -complete partial metric space, and suppose that $T: \bigcup_{i=1}^{k} A_{i} \rightarrow \bigcup_{i=1}^{k} A_{i}$ satisfies the following conditions (where $A_{k+1}=A_{1}$ ):

(1) $T\left(A_{i}\right) \subseteq A_{i+1}$ for $1 \leq i \leq k$;

(2) there exists $\alpha \in(0,1)$ such that $p(T(x), T(y)) \leq \alpha p(x, y) \forall x \in A_{i}, y \in A_{i+1}$ for $1 \leq i \leq k$. Then $T$ has a unique fixed point.

Proof One only needs to observe that given $x \in \bigcup_{i=1}^{k} A_{i}$, infinitely many terms of the Cauchy sequence $\left\{T^{n}(x)\right\}$ lie in each $A_{i}$. Thus $\bigcap_{i=1}^{k} A_{i} \neq \emptyset$, and the restriction of $T$ to this intersection is a contraction mapping.

Remark 17 It is our belief that Theorem 12 can be extended to more general cyclical contraction mappings. However, it would be of more interest if the contractive type conditions are considered with control functions.

The following theorem is an extension of an Edelstein type theorem to partial metric spaces.

Theorem 18 Let $\left\{A_{i}\right\}_{i=1}^{k}$ be nonempty closed subsets of a partial metric space $(X, p)$, at least one of which is 0-compact, and suppose that $T: \bigcup_{i=1}^{k} A_{i} \rightarrow \bigcup_{i=1}^{k} A_{i}$ satisfies the following conditions (where $A_{k+1}=A_{1}$ ):

(1) $T\left(A_{i}\right) \subseteq A_{i+1}$ for $1 \leq i \leq k$;

(2) $p(T(x), T(y))<p(x, y) \forall x \in A_{i}, y \in A_{i+1}$ for $1 \leq i \leq k$.

Then $T$ has a unique fixed point. 
Proof Assume $A_{1}$ is 0-compact, and let $\delta=p\left(A_{a}, A_{k}\right)=\inf \left\{p(x, y): x \in A_{1}, y \in A_{k}\right\}$. From the definition of $\delta$ there exist sequences $\left\{x_{n}\right\} \subset A_{1}$ and $\left\{u_{n}\right\} \subset A_{k}$ such that

$$
p\left(x_{n}, u_{n}\right) \leq \delta+\frac{1}{n}
$$

By 0 -compactness of $A_{1}$, we may assume that there exists $x_{0} \in A_{1}$ such that $\lim _{n \rightarrow \infty} p\left(x_{n}\right.$, $\left.x_{0}\right)=p\left(x_{0}, x_{0}\right)=0$. Then by the triangle inequality it follows that $\lim _{n \rightarrow \infty} p\left(x_{0}, u_{n}\right)=\delta$. Assume $\delta>0$. Then

$$
p\left(T^{k+1}\left(x_{0}\right), T^{k+1}\left(u_{n}\right)\right)<\cdots<p\left(x_{0}, u_{n}\right) .
$$

Since the sequence $\left\{T^{k+1}\left(u_{n}\right)\right\}$ is in $A_{1}$ and $A_{1}$ is 0 -compact, we may assume that there exists $z \in A_{1}$ such that $\lim _{n \rightarrow \infty} p\left(T^{k+1}\left(u_{n}\right), z\right)=p(z, z)=0$. By (5) and Lemma 10, we conclude that

$$
p\left(z, T^{k+1}\left(x_{0}\right)\right) \leq \delta .
$$

However, this implies

$$
p\left(T^{k-1}(z), T^{2 k}\left(x_{0}\right)\right)<\delta,
$$

and since $T^{k-1}(z) \in A_{k}$ and $F^{2 k}\left(x_{0}\right) \in A_{1}$, we have a contradiction. Therefore, we conclude that $\delta=0$ and $A_{1} \cap A_{k} \neq \emptyset$. Thus, by the assumption (1), $A_{1} \cap A_{2} \neq \emptyset$.

We now consider the sets $B_{1}=A_{1} \cap A_{2}, B_{2}=A_{2} \cap A_{3}, \ldots, B_{k}=A_{k} \cap A_{1}$. In view of the condition (1) these sets are all nonempty (and closed) and $B_{1}$ is 0 -compact. Thus the assumptions (1) and (2) of the theorem hold for $T$ and the family $\left\{B_{i}\right\}_{i=1}^{k}$. By repeating the argument just given, we arrive at

$$
B_{1} \cap B_{k} \neq \emptyset .
$$

This, in turn, implies that $A_{1} \cap A_{2} \cap A_{3} \neq \emptyset$. Continuing step-by-step, we conclude that $A:=\bigcap_{i=1}^{k} \neq \emptyset$.

Uniqueness follows from the fact that any fixed point of $T$ necessarily lies in $A:=\bigcap_{i=1}^{k}$ by the assumption (1).

\footnotetext{
Competing interests

The authors declare that they have no competing interests.
}

Authors' contributions

All authors contributed equally and significantly in writing this paper. All authors read and approved the final manuscript.

\section{Author details}

'Department of Mathematics, Çankaya University, Ankara, 06530, Turkey. ${ }^{2}$ Department of Mathematics and Physical Sciences, Prince Sultan University, P.O. Box 66833, Riyadh, 11586, Saudi Arabia. ${ }^{3}$ Department of Mathematics, University of Wisconsin-Fox Valley, Menasha, WI 54952, USA 


\section{References}

1. Khan, MS, Sweleh, M, Sessa, S: Fixed point theorems by alternating distance between the points. Bull. Aust. Math. Soc 30(1), 1-9 (1984)

2. Rhoades, BE: Some theorems on weakly contractive maps. Nonlinear Anal., Theory Methods Appl. 47(4), 2283-2693 (2001)

3. Dutta, PN, Choudhury, BS: A generalization of contraction principle in metric spaces. Fixed Point Theory Appl. 2008, Article ID 406368 (2008)

4. Boyd, DW, Wong, SW: On nonlinear contractions. Proc. Am. Math. Soc. 20, 458-464 (1969)

5. Matthews, SG: Partial metric topology. Research report 212, Department of Computer Science, University of Warwick (1992)

6. Matthews, SG: Partial metric topology. In: General Topology and Its Applications. Proc. 8th Summer Conf., Queen's College, 1992. Annals of the New York Academy of Sciences, vol. 728, pp. 183-197 (1994)

7. Abdeljawad, T, Karapinar, E, Taş, K: Existence and uniqueness of a common fixed point on partial metric spaces. Appl. Math. Lett. 24(11), 1900-1904 (2011)

8. Abdeljawad, T, Karapinar, E, Taş, K: A generalized contraction principle with control functions on partial metric spaces. Comput. Math. Appl. 63(3), 716-719 (2012)

9. Abdeljawad, T: Fixed points for generalized weakly contractive mappings in partial metric spaces. Math. Comput. Model. 54(11-12), 2923-2927 (2011)

10. Oltra, S, Valero, O: Banach's fixed point theorem for partial metric spaces. Rend. Ist. Mat. Univ. Trieste 36(1-2), 17-26 (2004)

11. Valero, O: On Banach fixed point theorems for partial metric spaces. Appl. Gen. Topol. 6(2) 229-240 (2005)

12. Altun, I, Sola, F, Simsek, H: Generalized contractions on partial metric spaces. Topol. Appl. 157(18), $2778-2785$ (2010)

13. Altun, I, Erduran, A: Fixed point theorems for monotone mappings on partial metric spaces. Fixed Point Theory Appl. 2011, Article ID 508730 (2011). doi:10.1155/2011/508730

14. Shatanawi, W, Samet, B, Abbas, M: Coupled fixed point theorems for mixed monotone mappings in ordered partial metric spaces. Math. Comput. Model. (2012). doi:10.1016/j.mcm.2011.08.042

15. Ilić, D, Pavlović, V, Rakočević, V: Some new extensions of Banach's contraction principle to partial metric spaces. Appl. Math. Lett. 24, 1326-1330 (2011)

16. Romaguera, S: A Kirk type characterization of completeness for partial metric spaces. Fixed Point Theory Appl. 2010 Article ID 493298 (2010). doi:10.1155/2010/493298

17. Kirk, WA, Srinivasan, PS, Veeramani, P: Fixed points for mappings satisfying cyclical contractive conditions. Fixed Point Theory 4(1), 79-89 (2003)

doi:10.1186/1687-1812-2012-154

Cite this article as: Abdeljawad et al.: Banach contraction principle for cyclical mappings on partial metric spaces. Fixed Point Theory and Applications 2012 2012:154

\section{Submit your manuscript to a SpringerOpen ${ }^{\circ}$ journal and benefit from:}

- Convenient online submission

- Rigorous peer review

- Immediate publication on acceptance

- Open access: articles freely available online

- High visibility within the field

- Retaining the copyright to your article 